ÉGYPTE monde arabe

\section{Égypte/Monde arabe}

$3 \mid 2000$

La censure ou comment la contourner

\title{
Le texte et la marge
}

Critique des mythes de l'Ancien Testament chez 'Isâm al-Dîn Hifnî Nâsif

\section{Anwar Moghith}

\section{(2) OpenEdition}

\section{Journals}

Édition électronique

URL : https://journals.openedition.org/ema/791

DOI : 10.4000/ema.791

ISSN : 2090-7273

Éditeur

CEDEJ - Centre d'études et de documentation économiques juridiques et sociales

Édition imprimée

Date de publication : 30 juin 2000

Pagination : 85-104

ISBN : 2-87027-856-X

ISSN : $1110-5097$

Référence électronique

Anwar Moghith, « Le texte et la marge », Égypte/Monde arabe [En ligne], 3 | 2000, mis en ligne le 08 juillet 2008, consulté le 07 juillet 2022. URL : http://journals.openedition.org/ema/791 ; DOI : https:// doi.org/10.4000/ema.791

Ce document a été généré automatiquement le 7 juillet 2022.

Tous droits réservés 


\title{
Le texte et la marge
}

\author{
Critique des mythes de l'Ancien Testament chez 'Isâm al-Dîn Hifnî Nâsif
}

\author{
Anwar Moghith
}

\section{NOTE DE L'ÉDITEUR}

Traduit de l'arabe par Samia Rizk

1 Le statut conféré aux mythologies dans la culture égyptienne contemporaine diffère selon que cette dernière est savante ou profane, et en fonction de l'origine des mythes en question. Ainsi, au niveau profane, la mythologie grecque évoque-t-elle beauté, amour et aventure, la mythologie indienne, une profonde sagesse, nous introduisant directement dans le domaine de la littérature et imposant, par conséquent, une approche esthétique. Les mythes de l'Égypte pharaonique sont, quant à eux, abordés le plus souvent dans un cadre de réappropriation du passé historique, de découverte des profondeurs de «l'âme égyptienne » et d'interprétation de nombreuses pratiques folkloriques actuelles. En revanche, il suffit d'utiliser l'expression «mythes de l'Ancien Testament " pour se retrouver dans un espace conflictuel dominé par les affrontements politiques, religieux et idéologiques, où règnent le jeu de l'accusation et de la dénonciation, et celui des procès d'intention et des stratégies discursives.

2 Au niveau savant, si les mythologies égyptienne, grecque et indienne sont perçues comme telles et font l'objet d'une approche herméneutique qui les considère comme des récits symboliques, les textes de l'Ancien Testament sont repris dans les ouvrages religieux et dans l'exégèse coranique comme des faits historiques quasiment dépourvus de toute dimension symbolique. C'est ainsi que la perspective critique moderne de ces textes achoppera de manière récurrente sur la question de l'historicité mise en avant par l'exégèse coranique.

Dans les sociétés de culture arabo-islamique, cette tension à l'œuvre dans le rapport à l'Ancien Testament ne date pas d'hier. L'Ancien Testament constitue, en effet, une source précieuse pour l'interprétation du Coran puiqu'il fournit une foule de détails sur l'histoire des prophètes, relatée sous une forme concise dans le Coran. Mais la tradition 
islamique veut que ce texte, considéré d'inspiration divine, ait été falsifié. Cette thèse ouvre la porte à des critiques d'ordre théologique ${ }^{1}$ comme à des critiques d'ordre rationnel ${ }^{2}$.

Toutefois, la critique moderne est différente. Née d'une période où la perception religieuse de l'univers est remise en question, elle porte non pas sur les différences entre le judaïsme et l'islam, mais sur ce que les deux religions ont en commun. Considéré comme nécessaire au processus de modernisation, ce type de critique a été porté par nombre de penseurs réformistes de l'époque de la Nahda (renaissance) qui avaient été influencés par la pensée du siècle des Lumières. Al-Tahtâwî évoque l'existence, chez les Français, de théories contraires à celles des livres saints mais qui sont indispensables au progrès de la science. Shiblî Shumayyil et Salâma Mûsâ expriment clairement leur penchant positiviste lorsqu'ils affirment que l'ère de la science est venue remplacer cette perception religieuse de l'univers. Cette remise en question n'a pas été sans susciter des polémiques. Rappelons à ce propos l'ouragan déchaîné par une simple allusion de Taha Husayn dans son ouvrage De la poésie antéislamique où il dit : « La Bible peut nous parler d'Abraham et d'Ismaël ; le Coran peut nous en parler également. Cependant, l'occurrence de ces deux noms, et dans la Bible et dans le Coran, ne suffit pas à prouver qu'ils ont réellement existé, ni à prouver la véracité historique du récit du voyage d'Ismaël à La Mecque. » (Husayn, 1925, p. 31) Le tollé fut ici provoqué par la remise en cause d'une croyance bien ancrée chez les musulmans, celle issue d'une lecture littéraliste selon laquelle tous les personnages et tous les événements évoqués dans le Coran ont réellement existé. Si donc certains penseurs affichent ouvertement leur athéisme ou estiment que les croyances scientifiques doivent se substituer aux croyances religieuses, rares sont ceux qui le font en affrontant les croyances fortement enracinées et en soumettant les récits religieux à une critique méthodique.

D'où l'intérêt tout à fait particulier de la critique des mythes de l'Ancien Testament dans l'œuvre de 'Isâm al-Dîn Hifnî Nâsif (1898-1969), dont il faudra s'employer à déterminer le statut. En s'aventurant, en effet, dans la critique des textes de l'Ancien Testament, ce dernier vise de manière indirecte les croyances des musulmans tout en essayant d'échapper aux pièges de la censure, officielle et populaire. Si cette critique indirecte des croyances islamiques s'inscrit, comme on le verra, dans la perspective du tanwîr (littéralement, éclairement), soit dans la postérité revendiquée du siècle des Lumières, reste qu'il faudra déterminer en quoi la critique directe des mythes de l'Ancien Testament est, elle, l'héritière de certaines traditions exégétiques et jusqu'à quel point elle est tributaire de déterminants idéologiques. Avant de s'atteler à cette tâche, on s'emploiera d'abord à situer l'auteur et son œuvre dans leur contexte historique.

Isâm al-Dîn Hifnî Nâsif Rationalisme, Marxisme et démocratie bourgeoise

6 Issu d'une famille qui a marqué de son empreinte la culture égyptienne contemporaine $^{3}$, Isâm al-Dîn Hifnî Nâsif est exemplaire de cette génération d'intellectuels engagés qui prend son essor dans les années qui précèdent et accompagnent l'indépendance de l'Égypte en 1922. La période d'intense activité intellectuelle de Nâsif se situe dans l'entre-deux-guerres. Après avoir adhéré au Parti national, il obtient une bourse et part en Allemagne pour étudier l'agronomie. Devenu marxiste, il participe, à son retour, aux débats intellectuels du pays; à travers l'activisme politique, il cherche à jouer un rôle dans la promotion de la culture 
scientifique, la modernisation de la société égyptienne (al-Nushû' wa-l-irtiqấ, 1928) et la réalisation de la justice sociale (al-Tajdîd al-ijtimâĉे, 1930). Non-révolutionnaire, il voudrait concilier le marxisme et les valeurs de la démocratie bourgeoise (Mabâdi' alishtirâkiyya, 1933). C'est ainsi qu'il s'attelera à : $1^{\circ}$ ) la diffusion du savoir scientifique, considéré comme moyen de démystification. Il a, par exemple, tenté de vulgariser la théorie de l'évolution de Darwin et le matérialisme historique de Marx; $2^{\circ}$ ) la défense du socialisme, vu comme moyen de libérer le peuple égyptien du cercle vicieux de la pauvreté et de l'exploitation ; $3^{\circ}$ ) la défense des valeurs démocratiques, traduite par le refus de la dictature et la dénonciation du fascisme ; $4^{\circ}$ ) la fondation de partis légaux, tels que le Hizb al-fallâh (Parti du paysan) en 1930 et le Parti de la Réforme sociale en 1936, en lieu et place d'une adhésion aux organisations marxistes clandestines.

7 Si sa méfiance à l'égard de la classe ouvrière signe les limites de son adhésion au marxisme (le socialisme qu'il forge s'adresse principalement aux intellectuels), le lien qu'il établit entre progrès social et caducité des croyances religieuses trahit une foi entière dans le rationalisme scientifique : «La théorie de l'évolution s'est levée comme un soleil, dissipant l'obscurité dans laquelle baignaient des milliers d'objets. Auparavant, on ne justifiait pas les choses par leur cause directe, on se contentait de les rapporter à la volonté de Dieu et à sa sagesse. On n'expliquait pas les différents phénomènes, tout au plus les décrivait-on, comme on interprète un vers au moyen d'une métaphore au lieu d'en dégager le sens profond ou les intentions plus lointaines qu'il recèle. Ce n'est pas en recourant à la métaphysique que les divers phénomènes peuvent être éclaircis, mais en se référant à des notions qui nous sont connues, c'est-àdire, à des lois telles que celles de la physique et de la chimie. » (Nâsif, 1952, p. 1)

Dans les années quarante et par la suite, les marxistes égyptiens avaient, de manière générale, suivi les préceptes de Marx quant à la nécessité de passer de la « critique du ciel » à la "critique de la terre $»^{4}$. La dénonciation politique de la réalité sociale dominait leurs écrits. Chez cIsâm al-Dîn Hifnî Nâsif, au contraire, la critique du ciel s'impose comme un impératif dans le contexte culturel arabomusulman, et les modalités d'une telle entreprise, semée d'embûches, vont faire partie intégrante de l'entreprise elle-même.

La critique des mythes de l'Ancien Testament

9 C'est à partir de la moitié des années soixante que toutes les œuvres de Nâsif portent sur la critique des mythes de l'Ancien Testament. L'intérêt accordé à cette question remonte toutefois à ses premiers ouvrages. Dès 1952, il voudrait traduire un ouvrage du libre penseur anglais Robert Ingersoll : annoncé, avec la mention «sous presse », en quatrième de couverture de son ouvrage sur la théorie de l'évolution, Asbâab al-irtiyâa fí tanzîl al-kitâb al-muqaddass (Des raisons de douter de la révélation de la Bible) ${ }^{5}$ ne paraît pas, mais d'autres ouvrages sur la question sont publiés du vivant de l'auteur : en 1965, il traduit de l'anglais un ouvrage de Joseph Louis sur les méfaits de la circoncision ${ }^{6}$, et il compose un ouvrage qui reprend la thèse de la falsification historique du texte biblique (voir infra), intitulé Mihnat al-tawrât cala aydî al-yahûd (La Bible dénaturée par les juifs).

10 Les autres ouvrages: Silat al-qurân bi-l-yahûdiyya wa-l-masîhiyya (Rapports du Coran avec le judaïsme et le christianisme), traduction d'un ouvrage ${ }^{7}$ de l'orientaliste allemand Wilhelm Rudolph (1975); Mûsâ wa fircawn bayna alustûriyya wal-târîkhiyya (Moïse et Pharaon, entre la mythologie et l'histoire, 1975) ; al-Ustûra wa-l-wacî (Le mythe et la conscience, 1976); al-Yahûdiyya fil-caqîda wa-l-târîkh (Le judaïsme: dogme et histoire, 1977) ; al-Masîh bi-mafhûmin mucâsir (Le Christ d'un point de vue contemporain, 
1979), sont tous parus plus de cinq ans après la mort de l'auteur. Le premier et le dernier livres ont été publiés à Beyrouth, les autres ont été publiés par une maison d'édition, fondée pour l'occasion, conformément à la volonté de sa femme qui tenait à publier les œuvres posthumes de son mari.

11 Si les deux premiers ouvrages de Nâsif sont historiques, le premier s'attachant à l'histoire de Moïse, le deuxième à celle de certains prophètes - Abel et Caïn, Abraham, Joseph et Lot - ; le troisième met en évidence l'origine païenne du judaïsme et les traces de paganisme que l'on retrouve dans la Bible - nonobstant ses déclarations de foi monothéiste et son affirmation de la transcendance. Le quatrième, quant à lui, s'intéresse à la personnalité du Christ du point de vue des figures de l'incarnation divine dans la mythologie antique.

$12 \mathrm{Au}$ vu des préoccupations spécifiques déjà mentionnées et des intérêts historiquement déterminés de Nâsif, par ailleurs traducteur de littérature russe et allemande, il est relativement aisé de deviner les motifs qui l'ont poussé à entreprendre une critique des mythes de l'Ancien Testament. Reste que les déterminants d'une telle critique - mais aussi ses modalités - doivent être élucidés, si l'on veut pouvoir la jauger à l'aune de ses performances : dans quelle tradition exégétique cette entreprise au long cours - elle occupe les vingt dernières années de sa vie - s'inscrit-elle ? En quoi - et à quel prix est-elle dictée par des mots d'ordre idéologiques contemporains?

Nationalisme et histoire La réhabilitation de l'Égypte antique?

13 Né au cours de la deuxième moitié du XIX ${ }^{\text {e }}$ siècle, dans le contexte de la domination étrangère, le nationalisme égyptien vise à établir un État-nation. Il est accompagné par un courant de pensée qui essaye de forger une identité de l'Égypte indépendamment de son appartenance religieuse - argument clé du maintien de son annexion à l'Empire ottoman. Pour ce faire, il est, entre autres, amené à reconsidérer l'histoire de l'Égypte pharaonique, dont la perception jusque-là dominante était héritée de la tradition biblique et coranique.

Cherchant à réhabiliter l'histoire de l'Égypte ancienne, Nâsif reste l'héritier de cette première forme du nationalisme égyptien. Les mythes de l'Ancien Testament recueillis dans le Coran suscitant en effet une certaine hostilité à l'encontre de la civilisation pharaonique, l'auteur essaie de démontrer que, le conflit opposant Moïse à Pharaon nonobstant, ce dernier est présenté dans l'Ancien Testament comme un personnage courtois et clément, "qui ne refuse pas de s'entendre par voie de conciliation, même avec un étranger dont les gestes laissent à penser que c'est un prestidigitateur et un magicien" (Nâsif, 1975, p. 12). Nâsif fait ressortir par ailleurs la haine profonde et injustifiée que nourrissent le Dieu des juifs et leurs prophètes à l'égard des Égyptiens. Lorsqu'il évoque l'épisode au cours duquel Yahvé a exaucé la neuvième invocation de Moïse et qu'une obscurité profonde a recouvert l'Égypte entière pendant trois jours, il ajoute : "Moïse aurait pu profiter de ces ténèbres épaisses et emmener les enfants d'Israël où bon lui semble. Mais en agissant ainsi, il aurait privé son dieu inique de frapper nos ancêtres égyptiens de la dixième plaie [la mort des premiers-nés dans toutes les familles d'Égypte]. » (Nâsif, 1975, p. 56) À la fin d'un chapitre émouvant où il fait ressortir l'injustice à laquelle fut exposé le peuple égyptien, Nâsif conclut par une question: "Quel crime perpétré par le peuple égyptien pouvait-il lui valoir ces fléaux? " (Nâsif, 1975, p. 58) Bien que l'auteur affirme que rien de la sorte ne s'est jamais produit, il est toutefois amené à reconnaître, pour le dénoncer, ce sentiment d'agressivité à l'encontre des anciens Égyptiens: fait-il, dans ce paradoxe, œuvre de 
réhabilitation? Difficile de le penser, dans la mesure où l'histoire de l'Égypte ancienne faisant partie intégrante de l'histoire de Moïse, la critique de cette dernière - objectif de Nâsif - entraîne nécessairement une représentation positive de l'Égypte ancienne. De plus, cette « défense et illustration » n'apparaît nulle part ailleurs dans son œuvre. En revanche, les mythes de tous les peuples anciens y apparaissent comme des manifestations d'une phase primitive de la Raison, et la mythologie égyptienne ne s'en distingue ni par une supériorité interne ni par une plus grande sagesse.

De l'anti-sionisme anecdotique au psychologisme

15 À partir de la seconde moitié du XX siècle, dans le contexte du conflit israélo-arabe, le nationalisme égyptien, devenu pan-arabe, établit un lien entre la création de l'État d'Israël et les mythes de l'Ancien Testament. «La Bible qui se trouve entre nos mains, dit Nâsif, n'est que le livre des prétentions sionistes tracées par les rabbins pour inciter leur peuple à usurper la terre de Palestine à ses propriétaires arabes » (Nâsif, 1975, p. 1). En fait, tout ce que les Arabes ont dû endurer à cause du mythe de la Terre promise ne peut que les pousser à critiquer l'Ancien Testament. Nâsif puise-t-il dans l'antisionisme de rigueur une motivation supplémentaire pour se livrer à la critique des mythes de l'Ancien Testament? Cette inclusion ou cette évocation du contexte contemporain étant une occurrence quasi unique dans l'œuvre de Nâsif, nous pouvons en douter. Que dire, cependant, et comment qualifier les jugements de valeur de Nâsif et les accusations sans appel qu'il adresse aux juifs de la Bible?

16 Ailleurs dans Mûsâ wa fircawn, l'auteur, en effet, va se livrer à une investigation pour le moins étrange, qui s'appuie sur les ressorts du psychologisme pour rendre compte de la réalité historique et sociale des juifs bibliques. Ainsi décrit-il l'Exode comme « un récit sacerdotal qui révèle le désir d'agressivité qui les anime" (Nâsif, 1975, p. 18). La description des dix plaies d'Égypte sur laquelle Nâsif s'étend est l'occasion pour lui d'accuser les juifs de sadisme et de violence gratuite (Nâsif, 1975, p. 10). L'histoire de Lot qui offrit ses filles au peuple pour assouvir ses désirs sexuels lui permet de souligner leur phallocratie et leur misogynie : «Cela n'est pas étonnant ; la femme, aux yeux de ces barbares, n'était qu'une machine à enfanter. » (Nâsif, 1976, p. 53) L'histoire de Joseph démontre leur passion pour l'argent et pour le gain. Les guerres du roi David révèlent les sentiments qu'ils éprouvent à l'égard d'autrui et leur racisme : la seule justification des guerres opposant les enfants d'Israël à leurs ennemis cananéens résiderait dans l'ascendance de ces derniers, la malédiction de Noé s'étant abattue sur la descendance de son fils Cham - qui avait vu la nudité de son père (Nâsif, 1977, p. 170).

Malgré ces jugements indigents en eux-mêmes, non pertinents quant au propos et qui ne dépareraient pas, par leur dimension caricaturale, dans la littérature antisémite occidentale, nous écartons l'idée que ces représentations soient l'expression d'un tel antisémitisme chez Nâsif. Ce, pour de multiples raisons, internes et externes à l'œuvre en question. Tout d'abord, il faut rappeler ici que l'auteur n'émet aucun jugement négatif sur ses contemporains de confession juive. Bien au contraire, et dans l'enthousiasme général pour le fascisme qui se répand en Égypte au début des années quarante et qui voit dans ce dernier le moyen de se débarrasser de l'occupation britannique, Nâsif publie un livre qui évoque l'échec éventuel du fascisme et qui dénonce le nazisme en termes marxistes: sa politique à l'égard des juifs est ainsi décrite comme un moyen démagogique pour gagner les faveurs de la classe moyenne allemande, promise au plein-emploi après l'extradition des juifs (Nâsif, 1941, p. 43). Ensuite, l'idéologie sous-jacente à sa critique des mythes bibliques, reste, rappelons-le, 
une foi absolue dans la science, la laïcité, l'émancipation de la femme et l'humanisme autant de credos qui ne cadrent guère avec l'antisémitisme, élément souvent constitutif des idéologies réactionnaires. Enfin et surtout, les «juifs » de la Bible visés par Nâsif sont, et sans doute selon une lecture marxiste, non pas le peuple juif mais la seule "classe " des hommes de religion, idéologues-manipulateurs qui défendent leurs intérêts : en définitive, tout ce qui est écrit dans l'Ancien Testament n'est autre, selon Nâsif, que le produit de leurs affabulations, produites et soutenues afin de maintenir le peuple dans un état d'ignorance et de soumission. Pour étayer son analyse, Nâsif s'appuie ici sur des données de la critique historique de la Bible qui mettent en doute la réalité de plusieurs récits, l'existence historique de personnages et l'attribution de certains livres aux prophètes eux-mêmes.

Quelle tradition critique? La critique des « récits israélites » (al-isrâ’ ĥliyyât)

Isrẩ̂liyyât est le terme utilisé par les spécialistes des sciences coraniques pour désigner les récits israélites auxquels ont eu recours les exégètes du Coran dans le but hagiographique de compléter les récits de vie des prophètes, brièvement relatés dans le Coran. Ces emprunts sont justifiés par la distinction opérée entre ce qui est canonique (sharî) et ce qui est narratif (qisasî) dans le texte révélé. Or, bien qu'admis pour leur seule valeur narrative, ces récits hors-texte furent critiqués à travers les âges par des personnalités fort diverses - que ce soit par des jurisconsultes tels qu'Ibn Hanbal et Ibn Taymiyya ou par des penseurs tels qu'al-Jâhiz et Ibn Khaldoun.

Dans son acception moderne, ce terme revêt une signification négative. Parmi les auteurs contemporains œuvrant pour le renouveau de la pensée islamique, certains attribuent en effet aux récits israélites la responsabilité de certaines altérations qui seraient survenues dans la tradition islamique. Selon eux, il faut absolument " prévenir le lecteur des temps modernes du fait qu'une grande partie de ce que les exégètes rattachent à la tradition n'est en fait que la reprise de récits israélites mettant en valeur un point de vue biblique plus qu'islamique. Le lecteur doit donc faire preuve de perspicacité à la lecture des récits touchant à l'image des Arabes et, plus particulièrement, des Égyptiens, et à la représentation de la femme dans la tradition en général - cette représentation étant en parfaite contradiction avec celle qu'en donne le Coran. Ces récits israélites semblent avoir particulièrement influencé les doctrines exégétiques dans les questions relatives à la femme » (al-Sharqâwî, 1999, p. 77). Comme donc on suppose a priori que les croyances islamiques sont exactes et que les récits israélites ne sont que des ajouts parasites, l'épuration dogmatique s'impose comme la tâche à accomplir : «Il importe que tous nos savants s'unissent pour dépouiller notre patrimoine des récits qui y ont été insérés, qui sont venus s'y greffer, et ce afin de distinguer ce qui en relève et ce qui est conforme au Coran de ce qui y a été glissé à notre insu pour des raisons religieuses et culturelles diverses. » (ibid.)

Or chez Nâsif, rien de tel. S'il critique en quelques endroits de son œuvre al-Tabarî qui, de tous les exégètes classiques, tend le plus à s'attacher à la tradition judaïque, rien n'indique qu'il accorde aux croyances islamiques plus de crédibilité qu'aux croyances israélites, ainsi que nous le verrons plus loin.

Rationalisme et Lumières

21 La critique des récits israélites n'a donc jamais constitué autre chose que de simples allusions, rares et isolées, dans les écrits de Nâsif. En revanche, ce que l'on y trouve en permanence et de manière constante, c'est la défense du rationalisme vis-à-vis des mythes que les gens continuent à prendre pour la réalité. 

représente l'étape primitive; il apparaît dans l'opposition entre la conscience scientifique et la conscience mythique. Nâsif utilise tout le système de qualifications ayant servi aux philosophes des Lumières pour décrire le phénomène religieux: superstition, ignorance et intolérance. C'est ce qu'il s'évertue à prouver tout au long de son œuvre. Il présente une image négative de tous les prophètes, contrairement à la tendance dominante dans la tradition musulmane qui voudrait qu'on les respectent et qu'on les vénère. La prophétie, selon lui, est la fonction de personnes désœuvrées n'ayant aucune occupation : « Les enfants d'Israël passèrent par des périodes où, dit-il, la profession de prophète exerçait un attrait croissant et où le nombre de prophètes atteignit des centaines "; « Le roi d'Israël rassembla les prophètes au nombre d'environ quatre cents » (Le livre des Rois, I, 22, 6). À d'autres moments, le peuple étant moins enclin à écouter de telles balivernes, l'inspiration des prophètes tarissait ainsi que leurs visions: "En ce temps-là il était rare que Yahvé parlât, les visions n'étaient pas fréquentes" (Samuel, I, 3, 1), (Nâsif, 1979, p. 88). L'auteur raille également le fait de qualifier les prophètes de «bien-aimés de Dieu »: «Tous les amis de Dieu ont prouvé qu'ils avaient des qualités que les amis des hommes n'auraient pas été fiers d'acquérir » (Nâsif, 1976, p. 26). Ainsi, Abraham, le père commun aux trois religions révélées, qui abandonna la maison paternelle à l'âge de 75 ans, était un imposteur qui avait épousé sa demi-sœur et demandé à sa femme de s'associer à son mensonge en prétendant devant le pharaon qu'elle était sa sœur dans le seul but d'obtenir plus de bétel. "Sara était âgée de 70 ans lorsque Pharaon s'éprit d'elle, elle avait 90 ans lorsque le roi de Gérar s'éprit d'elle et qu'Abraham, son époux, la lui présenta. » (Nâsif, 1976, p. 39)

Lot était un misogyne, Joseph un accapareur qui profita de la famine pour dépouiller le peuple égyptien de ses richesses, Moïse un rancunier, un sadique et un frivole. David était ingrat, luxurieux et cruel, Salomon un apostat ayant abjuré la religion de ses pères, un polygame et un assoiffé de pouvoir. En revanche, cette critique morale disparaît chez Nâsif lorsqu'il parle du Christ. Il le présente cependant comme un amalgame conceptuel entre un prophète israélite et un dieu de l'antiquité païenne. Enfin, concernant Muhammad, Nâsif s'abstient de dire quoi que ce soit. Dictionnaire philosophique de Voltaire. On constate en effet une ressemblance frappante entre les portraits des prophètes tracés par Nâsif et ceux que dresse Voltaire de ces mêmes prophètes, une similitude dans les traits de caractère que l'un et l'autre déduisent des mêmes versets et une identité des objets de raillerie ou de révolte chez l'un et l'autre. À titre d'exemple, citons : l'âge d'Abraham lorsqu'il quitte la maison de son père, l'âge de Sara lorsque les rois s'éprennent d'elle, la passion de Joseph pour la femme d'un castrat, le lien entre al-rûh (l'âme) et al-rîh (le vent), la brutalité d'un dieu assoiffé de sang8. La seule différence notable chez les deux auteurs réside dans les détails : les portraits de Nâsif en comportent effectivement davantage.

Outre cette approche iconoclaste de la représentation courante des prophètes, Nâsif traite les récits de l'Ancien Testament relatifs à la création et à la genèse de l'univers comme étant des réponses à des questions que l'homme se pose pour comprendre le monde qui l'entoure. Ces réponses deviennent par conséquent fausses et mal fondées après les découvertes et les théories scientifiques modernes. Pour Nâsif, les croyances religieuses ne sont, en fin de compte, que le produit d'anciennes croyances païennes et les vestiges hérités d'une mentalité primitive et révolue.

Égypte/Monde arabe, 3 | 2000 

convergence de vue du traducteur et de l'auteur, ou pourquoi le traducteur a choisi de traduire cet ouvrage. C'est dans un autre passage de son livre Le mythe et la conscience que Nâsif critique Rudolph sur un point de détail concernant la place qu'occupe Abraham dans les trois religions en ces termes : «Certains Orientalistes européens se sont livrés à des aberrations en prétendant que la vénération pour Abraham dans le Coran est une action entreprise par le Prophète [Muhammad] après le refus des juifs de Médine de se rallier à son message. » (Nâsif, 1976, p. 33) Et il poursuit dans la marge : «Citons ce que dit Wilhelm Rudolph, professeur de théologie à l'université de Tübingen en Allemagne, sur le lien entre le Coran, le judaïsme et le christianisme: "Au début, Muhammad pensait qu'il prêchait ce que les juifs et Jésus avaient prêché avant lui. Mais lorsqu'il s'installa à Médine et fréquenta les gens du Livre (ahl al-kitâb), il en fut déçu. Comme ils n'ont pas cru en son message, il devait, pour faire face à leur opposition, reconstruire sa religion sur de nouvelles bases. Le personnage d'Abraham, vénéré aussi bien par les juifs que par les chrétiens et qui n'était pas le propre de l'une ou l'autre des deux religions, lui fut d'un grand secours puisque celui-ci avait vécu avant la révélation de la Torah et de l'Évangile... Et comme il n'avait à l'époque rien à reprocher ni aux juifs ni aux chrétiens, il appela l'islam la religion d'Abraham... puis déclara que la Ka cba avait été construite par Abraham et Ismaël".» Nâsif commente: "Telles sont, parmi d'autres, les opinions émises par méconnaissance de l'environnement arabe et dont la conséquence a été l'application de normes valables pour l'Europe à la presqu'île d'Arabie. » C'est la seule critique formulée par Nâsif le traducteur à l'adresse de l'orientaliste allemand, dont toutefois il ne dément aucune des autres thèses exposées dans l'ouvrage.

Les grandes traditions absentes

Fortement influencé par les Lumières, Nâsif l'est aussi de manière réductrice : le texte religieux apporte des réponses erronées aux questions de l'homme sur l'univers; il suffit qu'apparaisse la vérité scientifique pour que les croyances religieuses se trouvent rejetées et que la réponse exacte se substitue à la fausse réponse. Depuis Feuerbach jusqu'à Marx en passant par Nietzsche cependant, la critique allemande nous a appris que le phénomène religieux n'est pas seulement ou avant tout une aliénation cognitive mais bien une aliénation existentielle. Le phénomène religieux est donc complexe, les 
rapports de l'homme à la religion doublement déterminés. La leçon qu'adresse la critique allemande au siècle des Lumières réside dans l'accent mis sur cette complexité: il ne suffit pas de faire poindre la vérité scientifique pour que disparaissent les croyances religieuses. Le moins que l'on puisse dire, c'est que Nâsif le «marxiste » n'a pas retenu ce versant-là de la critique allemande. Chez lui, le mot mythe revêt un sens exclusivement négatif et la représentation mythique de l'univers, caractérisée par la barbarie et la naïveté, est rattachée à la représentation religieuse toutes deux étant qualifiées de primitives.

Mais l'apport de l'anthropologie structurale de Lévi-Strauss a mis en cause cette thèse européocentrée? - selon laquelle toute autre conception de l'univers, tout autre système de valeur que celui rationaliste et scientiste, est primitif et barbare. En fin de compte, le conscient mythique n'est autre que l'expression d'une structure extrêmement complexe, ayant ses codes, ses symboles et ses mécanismes propres non moins complexes que la structure du conscient moderne. Il n'est donc pas question de classement hiérarchique du plus simple au plus complexe, du primitif au civilisé.

31 Absente aussi du cadre théorique adopté par Nâsif, et pour cause, la tradition psychanalytique : si, pour cette dernière, le phénomène religieux est lié au domaine de l'inconscient, pour les Lumières il représente, bien au contraire, un niveau primitif de la conscience.

Absente enfin la lecture herméneutique telle qu'élaborée par un Ricœur puisque précisément elle prend le contre-pied du rationalisme des Lumières dans ses rapports au texte religieux. Celui-ci ne saurait être pris dans son sens littéral comme s'il s'agissait d'un texte simple sans autres dimensions. Dans le texte religieux, nous nous trouvons en face d'une extraordinaire métaphore au-delà de laquelle il nous faut percevoir le vrai sens. Son discours ne passe pas par des mots désignant des objets, mais par des symboles. Il n'a pas, par conséquent, un sens fixe, déterminé et indépendant ; le sens est déterminé par l'effort fourni par le lecteur et sa participation, effort conditionné par les circonstances historiques et existentielles du lecteur luimême. C'est pourquoi le texte religieux reste toujours ouvert, générateur de nouveaux sens et donc, donnant lieu à de multiples interprétations qui se succèdent au fil du temps. Il est impensable qu'une interprétation déterminée d'un texte religieux puisse un jour être définitive.

33 Si la critique de Nâsif ne puise pas dans l'apport décisif de ces grandes traditions critiques, par tout ce qu'elle renferme d'explicite, d'implicite, mais surtout de non-dit, et à travers la contestation des croyances religieuses dominantes dans la culture arabe, elle est et reste donc essentiellement un appel au rationalisme. L'œuvre de cet auteur a joué en Égypte un rôle primordial pour cerner l'archéologie des croyances et a mené la pensée rationaliste dans des zones que peu de penseurs de la Nahda ont pu pénétrer. Lutter contre l'ignorance et l'exploitation, dénoncer l'aliénation dans la conscience mythique fut le fin mot - une conscience commune aux juifs, chrétiens et musulmans. Comment, par suite, la critique implicite, non frontale, non plus de la seule conscience, mais des croyances islamiques apparaittelle dans l'explicite de la critique de l'Ancien Testament ? C'est ce qu'un examen des occurrences du texte coranique et de leur statut dans l'œuvre de Nâsif nous montrera.

Le texte et la marge Le Coran dans le texte de Nâsif

Les références explicites. Bien que Nâsif n'évoque guère l'islam en tant que tel dans ses ouvrages, le texte coranique y jouit d'une présence notable et inhabituelle. On 
constate tout d'abord que cette présence dans le corps du texte se réduit à quelques citations limitées ou à des allusions et des renvois succincts. À titre d'exemple, le Coran est mentionné de manière explicite lorsqu'il s'agit de la place d'Abraham dans les trois religions : l'auteur cite alors les versets des trois textes sacrés, dont ceux du Coran: «Abraham n'était ni juif ni chrétien mais il était un vrai croyant soumis à Dieu; il n'était pas au nombre des polythéistes. » (Sourate III, 67) ; «Dieu a pris Abraham pour ami » (Sourate IV, 125) (Nâsif, 1976, p. 5).

La deuxième référence au Coran se trouve dans Le Christ d'un point de vue contemporain où Nâsif, avant de procéder à une mise en regard des textes coranique et biblique, évoque la première étape de l'Ancien Testament, celle où dominait une conception personnelle de Dieu. En manière d'introduction à cette juxtaposition, il écrit: " Autrefois, Yahvé entretenait avec ses prophètes des liens étroits sur des bases solides. Par exemple, la Bible raconte que Dieu a accompagné deux anges pour rendre visite à Abraham. Celui-ci les accueillit généreusement et c'est alors que Dieu lui annonça la naissance de son fils Isaac. » (Nâsif, 1979, p. 13) Sur une même page, Nâsif va citer, dans la colonne de gauche, Genèse, 18, versets $1,2,7,8,10,12,13,14,17,20$ et 22 et Genèse, 19, versets 1 et 16 ; et, dans la colonne de droite, la Sourate LI du Coran, versets 24 à 36 .

Genèse $18: 1,2,7,8,10,12,13,14,17,20$ et 22 .

1 -Yahvé lui apparut au Chêne de Mambré, tandis qu'il était assis à l'entrée de la tente, au plus chaud du jour.

2 -Ayant levé les yeux, voilà qu'il vit trois hommes qui se tenaient debout près de lui ; dès qu'il les vit, il courut de l'entrée de la tente à leur rencontre et se prosterna à terre. Il dit : Monseigneur, je t'en prie, si j'ai trouvé grâce à tes yeux, veuille ne pas passer près de ton serviteur sans t'arrêter.

7 -Puis Abraham courut au troupeau et prit un veau tendre et bon; il le donna au serviteur qui se hâta de le préparer.

8 -Il prit du caillé, du lait, le veau qu'il avait apprêté et plaça le tout devant eux; il se tenait près d'eux, sous l'arbre, et ils mangèrent.

10 -... alors, ta femme Sara aura un fils.

12 -Donc, Sara rit en elle-même, se disant: Maintenant que je suis usée, je connaîtrais le plaisir ! Et mon mari qui est un vieillard !

13 -Mais Yahvé dit...

14 -Y a-t-il rien de trop merveilleux pour Yahvé ?

17 -Yahvé s'était dit : Vais-je cacher à Abraham ce que je vais faire...

20 -Donc, Yahvé dit : Le cri contre Sodome et Gomorrhe est bien grand! Leur péché est bien grave.

22 -Les hommes partirent de là et allèrent à Sodome. Abraham se tenait encore devant Yahvé.

Genèse $19: 1,16$.

1 -Quand les deux anges arrivèrent à Sodome sur le soir...

16 -Et comme il hésitait, les hommes le prirent par la main, ainsi que sa femme et ses deux filles, pour la pitié que Yahvé avait de lui. Ils le firent sortir et le laissèrent en dehors de la ville.

Coran, LI : 24-36

24 -L'histoire des hôtes d'honneur d'Abraham ne t'est-elle pas parvenue?

25 -Ils entrèrent chez lui et lui dirent : «Salut ! »... Abraham dit : «Salut ! Ô gens inconnus!»

26 -Il alla discrètement trouver les siens puis il revint avec un veau gras.

27 -Il le leur présenta en disant : « Ne mangerez-vous pas?»

28 -Il avait peur d'eux. Ceux-ci dirent : «Ne crains pas! » et ils lui annoncèrent la bonne nouvelle d'un garçon instruit.

29 -Sa femme s'avança en criant; elle se frappait le visage et elle disait: "Une vieille femme stérile ?... » 
30 -Ils dirent : « Ton Seigneur a parlé ainsi ; il est, en vérité, le Sage, celui qui sait. »

31 -Abraham dit : Ô vous, les Envoyés ! Quelle est donc votre mission?»

32 -Ils dirent : « Nous sommes envoyés à un peuple criminel

33 -pour lancer contre eux des pierres d'argile

34 -marquées auprès de ton seigneur à l'intention des pervers. »

35 -Nous avons fait sortir de cette cité les croyants qui y demeuraient.

36 -Nous n'y avons trouvé qu'une seule maison habitée par des gens soumis à Dieu.

La juxtaposition de ces deux textes entreprise par Nâsif pourrait souligner ou bien la ressemblance qui est évidente, ou bien les différences. Par exemple, la personnification de Dieu est plus évidente dans le texte biblique que dans le texte coranique : si, dans le Coran, il est question d'»Abraham et de ses hôtes d'honneur", dans l'Ancien Testament Dieu lui-même est l'hôte d'Abraham. Mais, ni la ressemblance ni la différence ne suscitent aucun commentaire de la part de Nâsif. Pourtant, Nâsif introduit la juxtaposition des deux textes sacrés en disant: "Yahvé entretenait des liens étroits avec ses prophètes. » Cette introduction est valable pour ce qui concerne le texte biblique. Pour ce qui concerne le texte coranique, cette dernière s'interprète de plusieurs manières : ou bien elle indique les sources communes de deux textes; ou bien elle prétend que le texte coranique est une simple reproduction du texte biblique; ou bien encore elle montre l'évolution des représentations d'une même croyance ; ou bien enfin elle confirme la thèse qui domine les ouvrages de Nâsif, à savoir, la dénonciation de la mythologie comme manifestation d'irrationalité. Notons que ce procédé, qui consiste à mettre en regard les deux textes afin d'en faire ressortir les ressemblances, est utilisé par Wilhelm Rudolph dans l'ouvrage traduit par Nâsif, mais que l'orientaliste s'y ménage une place plus large pour ses propres commentaires.

Les allusions d'ordre linguistique. À côté des citations directes des versets coraniques dans son texte, Nâsif recourt à un autre moyen pour impliquer le texte coranique dans ces analyses : les allusions linguistiques. Ainsi utilise-t-il, ici ou là, des mots et des expressions propres au Coran. Évoquant les miracles de Yahvé, il dit : « Sur les ennemis des juifs, il lança des pierres d'argile », et cite ensuite l'Ancien Testament: "Yahvé lança du ciel sur eux d'énormes grêlons » (Josué, 10 : 11). Or le mot argile utilisé par Nâsif rappelle au lecteur le texte coranique où Dieu sauve la Kacba des agresseurs abyssiniens. Il en est de même lorsqu'il parle de Moïse devant Dieu. Dieu dit à Moïse : «Je suis, en vérité, ton Seigneur ! Ôte tes sandales : tu es dans la vallée sainte de Tuwa » (Sourate XX, 12). Puis, après ce verset coranique, Nâsif cite le texte biblique correspondant : « Dieu l'appela du milieu du buisson : Moïse, Moïse ! Ôte tes sandales de tes pieds, car le lieu que tu foules est une terre sainte.» (Exode, $3: 4$-5) (Nâsif, 1975, p. 32).

Qu'elles soient explicites ou allusives, les références au Coran sont rares. Le texte coranique se manifeste surtout en marge comme si l'auteur lui accordait peu d'importance.

Le Coran en marge du texte. Ainsi le Coran est-il rarement mentionné dans le corps du texte. En revanche, on trouve de multiples références aux versets coraniques en notes de bas de page. Lorsqu'il parle dans le corps du texte d'un événement précis ou d'une croyance déterminée, l'auteur fait figurer dans la marge le verset coranique correspondant. Par exemple, quand il évoque l'interdiction pour les juifs de consommer certains aliments, Nâsif cite un verset des Actes des apôtres : «Vous abstenir des viandes immolées aux idoles [et ici un numéro renvoyant aux notes], du sang, des chairs étouffées et de l'impudicité. » (Actes des Apôtres, 15 : 29) 

la bête morte, le sang, la viande de porc et tout animal sur lequel on aura invoqué un autre nom que celui de Dieu.» (Sourate II, 173) De même, dans son exposé sur la création de l'univers selon l'Ancien Testament, il cite Genèse (2:2) : «Dieu conclut au septième jour l'ouvrage qu'il avait fait et, au septième jour, il chôma, après tout l'ouvrage qu'il avait fait. » Dans la marge, nous trouvons le verset coranique suivant: « Nous avons créé en six jours les cieux, la terre et ce qui se trouve entre les deux, sans éprouver aucune fatigue.» (Sourate L, 38) (Nâsif, 1976, p. 5). Nous remarquons que les versets de l'Ancien Testament sont largement analysés et interprétés, alors que ceux du Coran ne font l'objet d'aucun commentaire.

41

Parfois, lorsqu'il s'agit de croyances propres aux Arabes et aux musulmans, l'auteur fait précéder les versets coraniques des expressions « les Arabes pensent » s'il est question de particularités linguistiques, ou "les musulmans pensent» dans le cas de particularités théologiques. Lorsqu'il parle des fils d'Adam, Abel et Caïn, il précise dans les notes que « les gens ont arabisé ces deux noms pour qu'ils riment et aillent de pair. Ainsi, Kâyin et Âbil deviennent Qâbîl et Hâbîl. Jésus (Yâsûc) devient Îsâa, pour rimer avec Mûsâ (Moïse) ; Saül et Goliath deviendront Tâlût et Gâlût, construits sur la même rime que Ya'gûg (Gog) et Ma'gûg (Magog), Hârût et Mârût (Nâsif, 1976, p. 6). Toutes ces expressions se trouvent dans le Coran et il est à noter que cette glose est empruntée à l'ouvrage de Wilhelm Rudolph cité plus haut, sans que cela ne soit mentionné par Nâsif. Cela se reproduit à propos de l'origine historique du «mythe » de l'enfer (géhenne) où il rapporte que les fils de Juda « ont construit le haut lieu de Tophèt dans la vallée de Ben-Hinnom, pour brûler leurs fils et leurs filles...» (Jérémie, $7: 31$ ). Dans les notes, il précise : «En hébreux, Ge-hinnom, devenu Gahannam en amharique a donné en arabe Guhannam (la Géhenne). Nom propre désignant une vallée proche de Jérusalem (actuellement Wâdî al-rabbânî), où les païens sacrifiaient leurs fils en offrande, ce nom désigne aujourd'hui le lieu, dans le ciel, destiné au supplice des damnés. » (Nâsif, 1977, p. 113)

Voulant démontrer que la prédilection que Jacob avait pour son fils Joseph était une " erreur dans sa manière de l'élever », car en agissant ainsi il allait susciter la rancune et la jalousie de ses frères à son égard, Nâsif cite le verset de l'Ancien Testament où Joseph, intériorisant cette supériorité, dit à son père : «J'ai encore fait un rêve : il me paraissait que le soleil, la lune et onze étoiles se prosternaient devant moi. » (Genèse, 37: 9) Mais, dans la marge, la démonstration-prétexte cède la place aux objectifs critiques, Nâsif s'y fendant d'une note hors de propos, avant de donner le verset coranique équivalent : "Dans la version anglaise [de la Bible], le mot utilisé est "star" qui veut dire étoile, alors que les planètes [kawkab, pl. kawâkib] sont les corps célestes qui tournent dans l'orbite d'une étoile. Cependant, les Arabes ne faisaient pas de distinction entre ces deux termes et les utilisaient indifféremment: Joseph dit à son père : “Ô mon père! j'ai vu onze kawkab ou étoiles, le soleil et la lune : oui, je les ai vus se prosterner devant moi”. » (Sourate XII, 4) (Nâsif, 1976, p. 71) Ici, Nâsif s'appuie sur une remarque linguistique pour suggérer une fois de plus l'intertextualité entre la Bible et le Coran.

Outre ces remarques sur l'intertextualité entre le Coran et la Bible Nâsif établit, toujours en marge du texte, des rapprochements d'ordre dogmatique. Il consacre, par exemple, un chapitre à la cause secrète de la sanctification et de l'occurrence du chiffre 7 dans l'Ancien Testament (cinq cents occurrences), puis il signale également les 
passages du Nouveau Testament où ce même chiffre est sanctifié. Il énumère ensuite : les sept anges chez les Chaldéens, les sept grands esprits chez les Persans, les sept péchés capitaux chez les Égyptiens, et enfin les sept portes de l'enfer (avec, en note, le verset suivant : « La Géhenne sera sûrement pour eux tous leur rendez-vous. Elle a sept portes : un groupe d'entre eux se tiendra devant chaque porte.» (Sourate XV, 43-44) Puis, dans le texte : « Les cieux sont sept et les terres sont sept » et dans les notes, il cite le verset: «Dieu est celui qui a créé les sept cieux et qui en a fait autant pour la terre.» (Sourate LXV, 12) (Nâsif, 1976, p. 79).

Certaines gloses comportent un plus large commentaire de la part de l'auteur. Ainsi, en parlant de la foi en la magie, Nâsif dit dans son texte : "Yahvé a clairement dit: "Ne laissez pas vivre une sorcière." C'est en vertu de ce saint verset que des milliers d'êtres humains ont perdu la vie, accusés de crimes qu'ils ne pouvaient pas avoir commis. Jusqu'au Moyen Âge, la sorcellerie occupait une place importante et exerçait une influence profonde. C'était une pratique exclusivement féminine et, aux yeux du clergé, les femmes sont des êtres "enclins au mal". » L'auteur renvoie ensuite à la marge où il dit que cette idée a été transmise aux Arabes, ce qui justifie le verset : « contre le mal de celles qui soufflent sur les nœuds» (Sourate CXIII, 4). Nâsif rapporte ici l'histoire relatée par les exégètes du Coran, selon laquelle la fille d'un Juif a voulu pratiquer la magie noire sur le prophète Muhammad, en faisant onze nœuds sur une corde. Après avoir raconté les détails de l'histoire tels que les relatent les exégètes, Nâsif commente en ces termes: «Nous avons recensé dans le Coran 63 occurrences du mot sorcellerie en plus des dérivés de ce mot : le mot djinns (al-jinn, al-jân, al-jinna) utilisé 39 fois, satan au singulier (al-shaytân) 70 fois, et au pluriel (shayâtîn) 18 fois. » (Nâsif, 1977, p. 31-32). Suivant la même logique, les passages où l'Ancien ou le Nouveau Testament sont cités renvoient, dans les notes, à des versets du Coran précédés parfois des mentions : "Les Arabes ont hérité de cette idée ", "Cette croyance nous a été transmise ", " Nous avons hérité de ce mythe », et autres expressions qui manifestent une volonté d'impliquer les versets coraniques dans le jugement porté sur les versets bibliques.

La critique des croyances islamiques. Selon la science des fondements de la religion (cilm usûl al-dîn), la définition du musulman est fondée sur la foi en Dieu, ses anges, ses prophètes, les livres révélés et l'au-delà. Partant du principe que les faits religieux appartiennent à la sphère des faits humains, Nâsif critique l'ensemble de ces croyances. Face à certains phénomènes religieux tels que la prophétie et les rituels, qu'il considère comme des points communs à toutes les religions, il oppose le rationalisme. À propos de Moïse, Nâsif dit : « Moïse, durant ces longues et dures années, escaladait de temps à autre la montagne pour rencontrer Yahvé et lui parler. Nous en avons connu d'autres qui avaient la même prédilection pour les montagnes comme lieu de rencontre avec leur Dieu, et au pied de ces montagnes, les lois célestes leur étaient révélées. » (Nâsif, 1975, p. 27) Nous sommes ici en présence d'une description d'un phénomène transreligieux, dépourvue de tout jugement de valeur. Cependant, ailleurs, l'auteur se livre à une critique acerbe de phénomènes irrationnels généralement associés aux religions. Toujours à propos de Moïse, après la rencontre avec Dieu : «Ainsi prend fin une étape importante de la vie de Moïse, celle des visions et des hallucinations, et commence une étape de démagogie, de magie et d'imposture, toutes choses dont s'accompagne toute religion. » (Nâsif, 1975, p. 47) dieu a sa spécificité. Les livres saints ne sont, à ses yeux, qu'une sorte de compilation 
des croyances primitives des peuples qui y croient. L'idée d'un au-delà a été écartée par sa diatribe contre l'idée de l'existence de l'âme, indépendante du corps et qui serait éternelle.

Les choses deviennent plus complexes lorsqu'il s'agit du concept de Dieu dont l'auteur ne nie pas l'existence. Son attaque devient particulièrement acerbe quand il aborde la question de la personnification de Dieu, ce Dieu qui dîne avec ses prophètes et les combat, qui exige de ses fidèles des sacrifices très chers pour exaucer leurs demandes, qui se délecte de l'odeur de l'holocauste. Pour Nâsif, la raison ne peut admettre cette acception païenne de Dieu. Il s'attaque également à l'idée d'un Dieu irritable qui, pris d'un accès de colère, peut supprimer des milliers d'innocents, un Dieu de menace et d'intimidation. "L'image de ce Dieu coléreux qui nous menace du doigt et nous ordonne en criant : ne faites pas ceci, ne faites pas cela et gare à qui fait cela, cette image est le plus grand obstacle à l'humanité qui cherche à se libérer de la peur et de l'ignorance et à se purifier l'esprit des mythes de ces barbares primitifs. » (Nâsif, 1977, p. 109)

La critique de ces croyances s'adresse, dans les commentaires de Nâsif, aux versets de l'Ancien Testament. Toutefois, si l'on regarde les versets coraniques qui leur sont juxtaposés dans le texte ou cités dans les notes, on se rend compte que le sens littéral des versets choisis par l'auteur s'oppose aux résultats de la science moderne et paraitt correspondre plutôt à des mythes légendaires. L'objectif de Nâsif est ici de limiter l'autorité épistémologique du Coran en y substituant le rationalisme moderne. Mais qu'il s'agisse de l'Ancien ou du Nouveau Testament ou du Coran, les versets sélectionnés par Nâsif ne représentent qu'une partie mineure de ces textes: la plupart des versets ayant une portée éthique, sociale et historique est laissée de côté. Il ne s'agit donc pas d'une critique systématique de ces livres. La critique de Nâsif ne vise pas les livres saints mais plutôt certaines croyances qui contredisent les vérités scientifiques. Les versets sélectionnés sont convoqués pour jouer ainsi le rôle de témoin de la présence de ces croyances.

La dialectique entre le texte et la marge. La plupart des versets coraniques sont cités en marge du texte et auraient pu ne pas figurer du tout sans pour autant nuire à la cohérence de celui-ci. Par ailleurs, les notes ne constituent pas en elles-mêmes un texte parallèle car si on les regarde isolément, on n'y trouve qu'un ensemble de versets dispersés, sans interprétation, sans commentaire, sans contexte. C'est peut-être là le lien naturel entre n'importe quel texte et ses notes : le texte forme un tout en soi et les notes en dépendent et n'ont pas d'autonomie propre. Normalement, les notes apportent des éclaircissements au texte pour faciliter l'accès au sens. Or, dans le cas de Nâsif, les notes ne sont pas destinées à expliciter le texte ou à en éclairer le sens mais l'inverse: on a le sentiment que le texte a été écrit pour expliciter les marges et en éclairer le sens et que, surtout dans cette forme dépourvue de tout commentaire et de tout jugement, ce sont elles qui orientent le texte, en déterminent la portée et les visées. L'auteur, très présent dans le texte par ses opinions, ses interprétations et les jugements catégoriques qu'il porte, est totalement absent dans les notes, laissant au lecteur le champ libre pour effectuer lui-même l'aller-retour entre le texte et les notes. Voltaire, qui, on l'a déjà dit, a exercé une grande influence sur Nâsif, ne dit-il pas : « Les livres les plus utiles sont ceux dont les lecteurs font eux-mêmes la moitié; ils étendent les pensées dont on leur présente les germes. » (cité dans Mervaud, 1994, p. 74) 
50 Nâsif que, vu le caractère sensible de la question, l'auteur recourt à une stratégie particulière du dit et du non-dit. "Le problème général de l'implicite est de savoir comment on peut dire quelque chose sans accepter pour autant la responsabilité de l'avoir dit, ce qui revient à bénéficier à la fois de l'efficacité de la parole et de l'innocence du silence. » (Ducrot, 1972, p. 12). Ducrot fait la différence entre l'implicite involontaire et l'implicite volontaire, ce dernier étant qualifié de "manœuvre stylistique ». Les citations marginales et systématiques de versets coraniques dans l'œuvre de Nâsif relèvent bien de ce second implicite - d'un acte volontaire et intentionnel s'inscrivant dans cette définition de Ducrot. Effectivement, ces manœuvres stylistiques « permettent au locuteur de susciter certaines opinions chez le destinataire sans prendre le risque de le formuler lui-même; elles permettent donc de faire croire sans avoir dit. Mais on demande souvent à l'implicite de répondre à une exigence beaucoup plus forte. Il ne s'agit pas de faire croire, il s'agit de dire, sans avoir dit. Or dire quelque chose, ce n'est pas seulement faire en sorte que le destinataire le pense, mais aussi faire en sorte qu'une de ses raisons de le penser soit d'avoir reconnu chez le locuteur l'intention de le lui faire penser. Et justement, il peut arriver qu'on souhaite à la fois dire (en ce sens fort), et pouvoir se défendre d'avoir voulu dire. En d'autres termes, il peut arriver que l'on veuille bénéficier à la fois de l'espèce de complicité inhérente au dire, et rejeter en même temps les risques attachés à l'explicite » (Ducrot, 1972, p. 15). En fin de compte, l'auteur se réserve un dernier alibi : le lecteur peut supposer que les versets coraniques sont cités dans la marge en guise de confirmation du récit, ou pour donner la version exacte correspondant à une version altérée, ou encore pour montrer l'évolution de la foi. L'auteur ne tranche jamais de manière explicite la question de ce choix. Le sens dépendra chez lui des allusions, des euphémismes et des métaphores et surtout, en premier et en dernier lieu, de l'application du lecteur. À la fin de son ouvrage Le Christ d'un point de vue contemporain, Nâsif dit : « Il est facile à l'époque actuelle de rattacher toutes les idées de l'Ancien et du Nouveau Testament à l'origine païenne dont elles s'inspirent. » (Nâsif, 1979, p. 154) Par contre, il ne dit rien à propos du Coran qui est omniprésent dans la marge de l'ouvrage.

\section{BIBLIOGRAPHIE}

Al-Sharqâwî cIffat, 1999, « al-Shakhsiyya al-yahûdiyya fî-l-tafsîr al-qur'ânî », Ibdhấ, nº 5, mai.

Ducrot Oswald, 1972, Dire et ne pas dire, Paris, Hermann.

Encyclopédie de l'islam, tome X, article «tahrîf ».

Husayn Taha, 1925, Fîl-l-shicr al-gâhilî, rééd. dans al-Qâhira, n 149, avril 1995.

Ibn Khaldoun, 1934, Prolégomènes, trad. M. de Slane, 3 tomes, Paris, Librairie orientale Paul Geuthner.

La Sainte Bible, 1961, traduite en français sous la direction de l'École biblique de Jérusalem, Paris, Éditions du Cerf. 
Le Coran (Essai d'interprétation du Coran inimitable), 1967, traduction par D. Masson, revue par Sobhi el-Saleh, Paris-Beyrouth, Gallimard/Pléiade-Dâr al-kitâb al-lubnânî.

Marx, 1982, Euvres III, La Pléiade, Paris, Gallimard.

Mervaud Christiane, 1994, « Le dictionnaire philosophique portatif », Revue internationale de Philosophie, $\mathrm{n}^{\circ} 1$.

Nâsif 'Isâm al-Dîn Hifnî :

- 1934, Ikhfâq al-fâshiyya, Le Caire, Matbacat lagnat al-ta'lîf wa-l-targama wa-l-nashr.

- 1952, Nazariyyat al-tatawwur, Le Caire, Matbcat Misr.

- 1975, Silat al-qur'ân bi-l-yahûdiyya wa-l-masîhiyya, traduction de l'allemand de l'ouvrage de Wilhelm Rudolph, Beyrouth, Dâr al-talîca.

- 1975, Mûsâ wa Fircawn, Le Caire, Dâr al-câlam al-gadîd.

- 1976, al-Ustûra wa-l-waĉ, Le Caire, Dâr al-câlam al-gadîd.

- 1977, al-Yahûdiyya fi-l-caqîda wa-l-tarîkh, Le Caire, Dâr al-câlam al-gadîd.

- 1979, al-Masîh fî mafhûm mucâsir, Beyrouth, Dâr al-talî́a.

Voltaire, 1964 (1764), Dictionnaire philosophique, Paris, Garnier Flammarion.

\section{NOTES}

1. Nous en trouvons une illustration historique exemplaire chez Ibn Hazm, lorsqu'il remarque dans al-Fasl fíl-milal que la Bible contient des contradictions, des impossibilités théologiques, des expressions anthropomorphiques de Dieu et des attributions de péchés aux prophètes. Voir, au sujet de la falsification, l'Encyclopédie de l'Islam, t. X, article " tahrîf».

2. Ainsi, dans sa célèbre Muqaddima, Ibn Khaldoun mentionne, par exemple, la surestimation par les juifs du nombre de soldats de l'armée de Moïse, qu'ils portent à six cent mille : « Masoudi et plusieurs autres historiens relatent (...) que Moïse, après avoir passé en revue les hommes en état de porter les armes et âgés de vingt ans et plus, trouve plus de six cent mille guerriers. » (Selon la bible, six cent trois mille cinq cent cinquante [Nomb. 1, 46].) Ibn Khaldoun élabore maints arguments contre cette prétention : a) une telle armée est difficile à entretenir et à manier ; b) les enfants de Jacob qui sont les premiers juifs entrés en Égypte étaient, selon la Bible, au nombre de 75. Comment peuvent-ils produire un si grand nombre d'hommes en trois générations (120 ans) ? ; c) Les armées des grands empires de l'époque dépassaient à peine cent mille guerriers ; et Ibn Khaldoun conclut : « Le renseignement fourni par les chroniques des Israélites, savoir, que la garde de Salomon se composait de douze mille fantassins et que sa cavalerie consistait en quatorze cents chevaux tenus au piquet devant les portes de son palais est le seul qui soit authentique ; quant aux contes populaires, on ne doit y avoir aucun égard. », (Ibn Khaldoun, 1934, p. 17)

3. Son père, poète et juriste, fut le fondateur de l'Académie de la langue arabe ; sa sœur, connue sous le pseudonyme de Bâhithat al-bâdiya, l'une des pionnières du mouvement d'émancipation de la femme, et son frère Magd al-Dîn, le traducteur, l'un des symboles du Parti national (al-Hizb al-watanî). 
4. Dans sa critique de la philosophie du droit chez Hegel, Marx dit : « C'est tout d'abord la tâche de la philosophie, qui est au service de l'histoire, de démasquer l'aliénation de soi dans ses formes profanes, une fois démasquée la forme sacrée de l'aliénation de l'homme. La critique du ciel se transforme ainsi en critique de la terre, la critique de la religion en critique du droit, la critique de la théologie en critique de la politique. » (Marx, 1982, p. 383). Ce texte est souvent utilisé pour justifier l'orientation des marxistes égyptiens qui consiste à donner la priorité à la critique de la réalité sociale. C'est oublier que Marx commence ainsi son ouvrage : « Pour l'Allemagne, la critique de la religion est pour l'essentiel achevée. » Pour l'Égypte, en revanche, la critique du ciel reste la tâche à accomplir.

5. N'ayant pas pu avoir accès à ce texte, nous n'avons pas su quel texte original d'Ingersoll est traduit sous ce titre. La consultation du fichier central de la Bibliothèque de France, qui fait apparaitre six titres de l'auteur anglais, nous invite à penser qu'il s'agirait peut-être de The Mistakes of Moses (s.l.n.d.).

6. Al-Khitân dalâlatun isrấ̂liyya mu'ziya (La circonsision, une aberration israélite nocive) de Joseph Lewis. Ici, nous n'avons pu identifier ni le titre original, ni l'auteur.

7. Ouvrage non identifié.

8. Ainsi lisons-nous dans le Dictionnaire philosophique (cité ici dans l'édition Flammarion de 1964) : « [Abraham] amène à Memphis sa femme Sara, qui était extrêmement jeune, et presque enfant en comparaison de lui, car elle n'avait que soixante et cinq ans. Comme elle était très belle, il résolut de tirer parti de sa beauté », p. 23 ; « La jeune Sara avait quatre-vingt-dix-neuf ans selon l'Écriture quand Dieu lui promit qu'Abraham, qui n'en avait alors que cent soixante, lui ferait un enfant dans l'année ", p. 24. Citant l'Ancien Testament, Voltaire rapporte : « Lorsque le Seigneur vous aura livré les nations, égorgez tout, sans épargner un seul homme et n'ayez aucune pitié de personne », p. 30 ; et il écrit : « David était alors à la tête de six cents bandits, il allait faire des courses chez les alliés de son Bienfaiteur Achis ; il pillait tout, il tuait tout, vieillards, femmes, enfants à la mamelle. Et pourquoi égorgerait-il les enfants à la mamelle?», p. 159 ; «David s'empare de tout le royaume ; il surprend la petite ville ou le petit village de Rabbath, et il fait mourir tous les habitants par des supplices assez extraordinaires ; on les scie à deux, on les déchire avec des herses de fer, on les brûle dans des fours à briques; manière de faire la guerre tout à fait noble et généreuse ", p. 159 ; «Cependant, la famine arriva comme Joseph l'avait prédit, et Joseph, pour mériter les bonnes grâces de son roi, força le peuple à vendre ses terres à Pharaon; et toute la nation se fit esclave pour avoir du blé : c'est là apparemment l'origine du pouvoir despotique », p. 247 ; « Est-il vraisemblable que, dans un désert où le peuple juif n'avait ni cordonnier, ni tailleur, et où le Dieu de l'univers était obligé de faire un miracle continuel pour conserver les vieux habits et les vieux souliers des juifs, il se soit trouvé des hommes assez habiles pour graver les cinq livres du Pentateuque sur le marbre ou sur le bois ? », p. 296 ; « Quoi ! le Dieu qui vous parle a égorgé pour nous faire plaisir, tous les premiers-nés d'Égypte et s'il y a dans ce pays-là trois cent mille familles, cela fait trois cent mille hommes morts en une nuit pour nous venger; et vous n'avez pas secondé votre Dieu ! ", p. 297 ; « Personne ne sait ce que c'est que l'être appelé esprit, auquel même vous donnez son nom matériel d'esprit, qui signifie vent ", p. 32. 
INDEX

Mots-clés : islam, religion, mythe, Ancien Testament, exégèse coranique, marxisme 